\title{
Serum Procalcitonin for Discrimination of Blood Contamination from Bloodstream Infection due to Coagulase-Negative Staphylococci
}

\author{
P. Schuetz, B. Mueller, A. Trampuz
}

\begin{abstract}
The diagnostic value of serum procalcitonin (PCT) to distinguish blood contamination from bloodstream infection (BSI) due to coagulase-negative staphylococci was evaluated. Patients with BSI had higher PCT concentration than those with blood contamination at day -1 , day 0 and day +1 with regard to blood culture collection $(p<0.05)$, whereas serum $C-$ reactive protein values were significantly higher only on day +1 . At a cutoff of $0.1 \mathrm{ng} / \mathrm{dl}$, PCT had a sensitivity of $86 \%$ and $100 \%$, and a specificity of $60 \%$ and $80 \%$ for the diagnosis of BSI on day -1 and 0 , respectively. In addition to clinical and microbiological parameters, PCT may help discriminating blood contamination from BSI due coagulase-negative staphylococci.
\end{abstract}

Infection 2007; 35: 352-355

DOI 10.1007/s15010-007-7065-0

\section{Introduction}

Coagulase-negative staphylococci as ubiquitous skin commensals are both, the most common cause of nosocomial bloodstream infection (BSI) and blood culture contamination [1-3]. It is important to distinguish blood contamination from BSI to promptly initiate an adequate therapy in BSI and, in contamination to avoid unnecessary antimicrobial usage and healthcare expenditures, reduce the selection pressure on microorganisms and prevent potential drug adverse events.

Early and accurate parameters to distinguish contaminated blood from BSI are lacking [4]. Serum procalcitonin (PCT) has emerged as a biomarker for diagnosis of various bacterial infections and was found to have higher diagnostic accuracy as compared to clinical characteristics or commonly used laboratory parameters, such as white blood count (WBC) and serum C-reactive protein (CRP) [5-9]. However, the diagnostic accuracy of PCT to distinguish blood culture contamination from BSI is not known. Therefore, we prospectively compared PCT values with currently used routine clinical, microbiological and laboratory parameters to predict BSI due to coagulase-negative staphylococci.

\section{Patients and Methods}

The study was conducted at the University Hospital Basel in Switzerland, a 950-bed tertiary healthcare center. We prospectively included consecutive patients $\geq 18$ years of age admitted to our hospital between 1 April and 31 August 2005, from whom at least one blood culture grew coagulase-negative staphylococci. Patients with one or more positive blood cultures collected within 10 days were considered as one episode. Patients with positive blood cultures from outside hospitals and those obtained postmortem were excluded from the analysis. Blood cultures were processed using an automated colorimetric detection system (BacT/ALERT, bioMerieux, Durham, NC, USA) [10]. The time to positivity was defined as the time between the start of incubation and the instrument signal indicating growth in either the aerobic or anaerobic blood culture bottle, confirmed by Gram staining.

Patient records were reviewed with a standardized datacollection case report form to retrieve demographic, clinical, microbiological, radiographic, and laboratory data, including systemic inflammatory response syndrome (SIRS) criteria, and the number of positive and the total number of collected blood cultures. Patients were classified by the attending physician and his or her supervisor on the ward using clinical judgment, routine laboratory results and Centers for Disease Control and Prevention (CDC) criteria for nosocomial infections [11]. BSI was diagnosed if at least two SIRS criteria were present and at least one of the following criteria was fulfilled: (i) $\geq 2$ positive blood cultures with coagulase-negative staphylococci or (ii) time to positivity was $<15 \mathrm{~h}$ after blood culture collection. An infectious disease specialist, who was not aware about the PCT values, prospectively classified episodes into (i) blood contamination due to coagulase-negative staphylococci, (ii) BSI due to coagulase-negative staphylococci (without other focus of infection) and (iii) other infection with concomitant isolation of coagulasenegative staphylococci in blood culture. The classification of the episodes was confirmed by an independent senior infectious

\footnotetext{
P. Schuetz, B. Mueller

Dept. of Internal Medicine, University Hospital Basel, Petersgraben 4, Basel 4031, BS, Switzerland

A. Trampuz (corresponding author)

Dept. of Infectious Diseases and Hospital Epidemiology, University

Hospital Basel, Petersgraben 4, Basel 4031, BS, Switzerland;

e-mail: trampuza@uhbs.ch
}

Received: January 29, 2007 - Accepted: March 13, 2007 Published online: September 19, 2007 
diseases consultant. Blood culture collection, microbiological tests and antibiotic therapy were prescribed by attending physicians per usual practice. The Institutional Review Board classified the study as a quality control study and waived the need for patient informed consent.

Laboratory analyses included the determination of WBC and CRP from the routinely collected blood analysis of all patients. CRP concentrations were determined by an enzyme immunoassay having a detection limit of $<5 \mathrm{mg} / \mathrm{dl}$ (EMIT, Merck Diagnostica, Zurich, Switzerland). To insure true blinding in regard to PCT values, blood samples were collected from laboratory stock at day -1 , day 0 and day +1 with regard to blood culture collection and stored at $-70{ }^{\circ} \mathrm{C}$. At the end of the study, PCT was determined as a batch analysis using an ultrasensitive immuno-luminometric assay having a functional detection limit of $<0.05 \mathrm{ng} / \mathrm{ml}$ (PCT sensitive LIA ${ }^{\circledR}$, Brahms, Berlin, Germany)

To evaluate differences between groups, the unpaired Student's t-test for normally distributed continuous variables and the Mann-Whitney U-test or Fisher's exact test for categorical variables was used, as appropriate. If CRP or PCT was not detectable, a value equal to the limit of detection for the assay was assigned. To compare the diagnostic value of individual laboratory markers for diagnosing BSI, we performed a receiver operating characteristic (ROC) analysis [12]. The area under the ROC curve (AUC) was the measure of the accuracy of laboratory parameters to distinguish both groups. A p-value $<0.05$ (for a 2-sided test) was considered statistically significant. All calculations were performed using statistical software MedCalc for Windows (version 7.2.1.0, Mariakerke, Belgium).

\section{Results}

A total of 40 patients (median age 63 years; range 1889 years, $65 \%$ men) were included; 12 were classified as blood contamination, 7 as BSI due to coagulase-negative staphylococci and 21 as having an infection other than due to coagulase-negative staphylococci. The underlying medical conditions included hematological and solid malignancies $(48 \%)$, cardiovascular disease $(28 \%)$, respiratory disease $(13 \%)$, gastrointestinal disease $(10 \%)$, renal failure (10\%) and drug abuse (15\%). The most probable reasons for fever in 12 patients with blood contamination were malignancy $(n=3)$, viral infection $(n=2)$, rheumatologic inflammatory disease $(n=1)$, or the reason was unknown $(n=6)$. All seven patients with BSI due to coagulase-negative staphylococci had vascular catheters inserted at the time of blood culture collection (five with central venous catheters, two with peripheral venous catheters). In three patients with BSI the catheter was removed and in two of them $>15$ colonies of coagulase-negative staphylococci grew on the removed catheter tip culture using the roll-plate method. In patients with underlying infection, pneumonia $(\mathrm{n}=12)$, urosepsis $(n=4)$, soft tissue infection $(n=3)$, endocarditis $(n=1)$, fever in neutropenia without pathogen identification $(n=1)$ were diagnosed. In this group, the following causative microorganisms were isolated: Streptococcus pneumoniae $(\mathrm{n}=4)$, Streptococcus pyogenes $(\mathrm{n}=1)$, Escherichia coli $(\mathrm{n}=3)$, Enterobacter cloacae $(\mathrm{n}=1)$ and no pathogen found $(\mathrm{n}=11)$. Empiric antimicrobial therapy received all 7 patients with BSI due to coagulasenegative staphylococci, 15 of 21 patients (71\%) with an underlying infection other than due to coagulase-negative staphylococci and 2 of 12 patients (17\%) with blood contamination.

At the time of blood culture collection, mean body temperature $\left( \pm\right.$ standard deviation) was $38.6{ }^{\circ} \mathrm{C}( \pm 1.0)$ in patients with BSI due to coagulase-negative staphylococci, $38.7^{\circ} \mathrm{C}\left( \pm 0.6^{\circ}\right)$ in patients with underlying infection and $37.9{ }^{\circ} \mathrm{C}( \pm 0.8)$ in patients with blood contamination. All patients with underlying infection had $>2$ SIRS criteria present, namely fever $(n=6)$, tachycardia $(n=4)$, tachypnea $(n=3)$, leukocytosis or leucopenia $(n=4)$. In patients with blood contamination one SIRS criterion was present in six patients [fever $(n=3)$, tachycardia $(n=2)$, leukocytosis or leucopenia $(n=1)]$; two SIRS criteria were present in three patients [fever $(\mathrm{n}=3)$, tachycardia $(\mathrm{n}=1)$, leukocytosis or leucopenia $(\mathrm{n}=2)]$.

Table 1 shows laboratory markers of the patients with blood contamination and BSI due to coagulase-negative staphylococci. Patients with BSI had significantly higher PCT at all time points (day -1 , day 0 and day +1 ) than those with blood contamination, whereas CRP values were significantly higher only on day +1 (Figure 1 ). The time to positivity was $<15 \mathrm{~h}$ for 6 of 7 patients determined to have BSI and for only 1 of 12 patients with blood contamination The proportion of positive blood culture bottles in patients with BSI was $67 \%$ (3.0 of 4.5 bottles) as compared to $34 \%$ ( 1.3 of 3.8 bottles) in those patients with blood contamination. In patients with underlying infection, laboratory parameters were influenced by the underlying infection and were therefore excluded in further comparisons.

As determined by the ROC curve analysis, PCT was an accurate blood marker for distinguishing BSI from blood contamination (Table 1). At any time points, namely at day -1 , day 0 and day +1 , the accuracy of PCT was significantly higher than the one of CRP $(\mathrm{p}<0.01)$. For PCT, the cutoff of $0.1 \mathrm{ng} / \mathrm{dl}$ had a sensitivity, specificity, positive and negative likelihood ratio of $86 \%, 60 \%$, 2.2 and 0.2 (at day -1), 100\%, 80\%, 6.0 and 0.1 (at day 0 ) and $100 \%, 84 \%, 4.2$ and 0.2 (at day +1 ), respectively.

\section{Discussion}

Previous studies have focused on finding a sensitive diagnostic marker for prediction of BSI and early guidance of antimicrobial therapy, however, accurate diagnostic criteria are still lacking [4]. Microbiological characteristics like capability of biofilm production, quantitative blood cultures, the proportion of positive blood cultures or the time to positivity of blood cultures has been suggested to be discriminative in this setting [4, 9, 13]. In Staphylococcus aureus, the time to positivity of blood cultures provided useful diagnostic and prognostic 


\begin{tabular}{|c|c|c|c|c|}
\hline Laboratory marker & $\begin{array}{l}\text { Time regarding to } \\
\text { blood collection }\end{array}$ & $\begin{array}{l}\text { Blood contamination } \\
(n=12)\end{array}$ & $\begin{array}{l}\text { Bloodstream } \\
\text { infection } \\
(n=7)\end{array}$ & $\begin{array}{l}\text { AUC }(95 \% \text { confidence } \\
\text { interval) })^{\mathrm{a}}\end{array}$ \\
\hline \multirow[t]{3}{*}{ WBC $\left(\times 10^{9} / l\right)^{b}$} & Day -1 & $9.3(0.1-11.9)$ & $11.1(1.1-25.0)$ & - \\
\hline & Day 0 & $8.6(0.1-12.0)$ & $11.5(0.5-28.0)$ & - \\
\hline & Day +1 & $6.3(0.1-12.0)$ & $16.0(0.5-31.0)$ & - \\
\hline \multirow[t]{3}{*}{ CRP (mg/dl) } & Day -1 & $48(4-207)$ & $48(16-278)$ & $0.56(0.38-0.80)$ \\
\hline & Day 0 & $30(4-193)$ & $64(47-417)$ & $0.67(0.42-0.87)$ \\
\hline & Day +1 & $45(4-147)$ & $168(36-212)$ & $0.80(0.54-0.96)$ \\
\hline \multirow[t]{3}{*}{ PCT (ng/ml) } & Day -1 & $0.09(0.05-0.18)$ & $0.50(0.06-5.27)$ & $0.87(0.62-0.98)$ \\
\hline & Day 0 & $0.08(0.05-0.23)$ & $0.62(0.14-5.20)$ & $0.98(0.78-0.98)$ \\
\hline & Day +1 & $0.08(0.05-0.20)$ & $0.55(0.15-2.70)$ & $0.97(0.74-0.98)$ \\
\hline Time to positivity of blood cultures $(h)^{b}$ & & $19.1(6.7-27.5)$ & $10.7(3.9-29.7)$ & - \\
\hline No. positive blood culture bottles/no. total & & $1.3(1-2) / 3.8(2-8)$ & $3.0(1-6) / 4.5(2-6)$ & - \\
\hline
\end{tabular}

information; growth within 12-14 $\mathrm{h}$ after the initiation of incubation identified patients with a high likelihood of endovascular infection sources, delayed bacterial clearance and septic complications [14]. However, the time delay of several days and the financial cost are major disadvantages of this approach.

Commonly used blood markers such as WBC and CRP may be elevated, but often reflect the nonspecific systemic inflammatory response to the underlying disease state rather than to BSI. In this study, PCT at a low cutoff of $0.1 \mathrm{ng} / \mathrm{ml}$ was an early and accurate laboratory parameter to distinguish BSI from blood contamination due to coagulase-negative staphylococci, which was superior to the accuracy of CRP. These findings are consistent with other reports in comparable settings, showing that PCT is a more reliable parameter for distinguishing infectious from non-infectious febrile episodes and for initiation and guidance of antimicrobial treatment $[5-8,15]$. Our findings also underline the importance of the use of more sensitive PCT assays to appreciate its full diagnostic potential [16].

An important finding of our study was the increased values of PCT 1 day prior to clinical manifestation of BSI and collection of blood cultures. The early increase of PCT may reflect the colonization of the catheter with subclinical infection ultimately leading to BSI [17]. CRP was discriminative only at 1 day after blood culture collection; even at this point, PCT had a superior diagnostic accuracy. Since contamination due to coagulase-negative staphylococci is occurring in approximately $23 \%$ of all collected blood cultures $[1,4,18]$, correct evaluation of their significance is important.
Limitations of this exploratory study are the small sample size and the heterogeneity of patients regarding their underlying diseases and the cause of febrile episodes. In patients with polymicrobial BSI, PCT may be of limited value to diagnose BSI caused by coagulasenegative staphylococci, since the additional microorganism(s) may be responsible for the increased PCT concentration. PCT values need to be evaluated with caution in neutropenic patients since the course of the bacterial infection can be overwhelmingly fast in neutropenia devoid of an efficient host defense [6]. Furthermore, the specificity of PCT may be limited in patients with lung and thyroid cancer [19]. Therefore, our results need to be confirmed in larger studies. Another limitation relates to the observed time to positivity which may be affected by different time from the blood collection until incubation, since bacterial growth at room temperature $\left(22{ }^{\circ} \mathrm{C}\right)$ is considerably slower than at incubator temperature $\left(37^{\circ} \mathrm{C}\right)$.

In conclusion, if confirmed in larger studies, PCT may serve as an early and more accurate surrogate marker for differentiation of BSI from blood contamination due to coagulase-negative staphylococci, especially when used as an adjunct to clinical, other laboratory and microbiological parameters. Early identification of patients with blood contamination has great clinical relevance for an improved diagnostic approach and to prevent unnecessary antimicrobial treatment. However, determination of PCT should never substitute a careful history, physical examination and evaluation of other laboratory parameters, including time to positivity of blood cultures. 

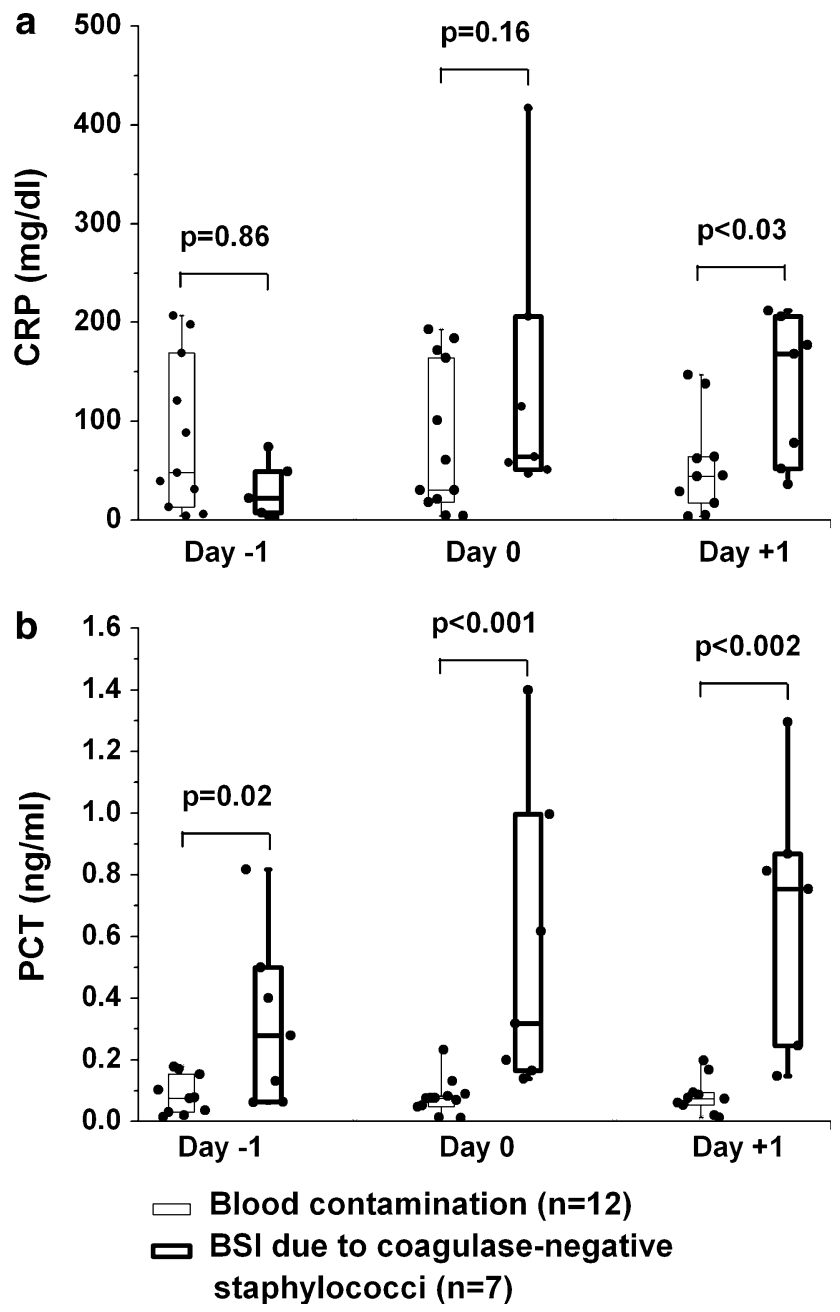

Figure 1. CRP (a) and PCT (b) values in patients with blood contamination (left side, fine lines) and bloodstream infection (right side, strong lines) the day before (day -1), the day of (day o) and the day after (day +1$)$ blood culture collection. Horizontal lines in boxes denote median values, boxes represent 25 and 75 percentiles and whiskers indicate the range. Values are missing for two subjects on day -1 and for three subjects on day +1 .

\section{Acknowledgments}

This study was in part presented at the 16th European Congress of Clinical Microbiology and Infectious Diseases, Nice, France, 1-4 April 2006 (Abstract O-217).

\section{References}

1. Thylefors JD, Harbarth S, Pittet D: Increasing bacteremia due to coagulase-negative staphylococci: fiction or reality? Infect Control Hosp Epidemiol 1998; 19: 581-589.

2. Kirchhoff LV, Sheagren JN: Epidemiology and clinical significance of blood cultures positive for coagulase-negative staphylococcus. Infect Control 1985; 6: 479-486.
3. Edmond MB, Wallace SE, McClish DK, Pfaller MA, Jones RN, Wenzel RP: Nosocomial bloodstream infections in United States hospitals: a three-year analysis. Clin Infect Dis 1999; 29: 239-244.

4. Garcia P, Benitez R, Lam M, et al. Coagulase-negative staphylococci: clinical, microbiological and molecular features to predict true bacteraemia. J Med Microbiol 2004; 53: 67-72.

5. Simon L, Gauvin F, Amre DK, Saint-Louis P, Lacroix J: Serum procalcitonin and C-reactive protein levels as markers of bacterial infection: a systematic review and meta-analysis. Clin Infect Dis 2004; 39: 206-217.

6. de Bont ES, Vellenga E, Swaanenburg J, Kamps W: Procalcitonin: a diagnostic marker of bacterial infection in neutropenic cancer patients with fever? Infection 2000; 28: 398-400.

7. von Lilienfeld-Toal M, Dietrich MP, Glasmacher A, et al. Markers of bacteremia in febrile neutropenic patients with hematological malignancies: procalcitonin and IL- 6 are more reliable than C-reactive protein. Eur J Clin Microbiol Infect Dis 2004; 23: 539-544.

8. Christ-Crain $M$, Jaccard-Stolz D, Bingisser R, et al. Effect of procalcitonin-guided treatment on antibiotic use and outcome in lower respiratory tract infections: cluster-randomised, single-blinded intervention trial. Lancet 2004; 363: 600-607.

9. Haimi-Cohen Y, Vellozzi EM, Rubin LG: Initial concentration of Staphylococcus epidermidis in simulated pediatric blood cultures correlates with time to positive results with the automated, continuously monitored BACTEC blood culture system. J Clin Microbiol 2002; 40: 898-901.

10. Thorpe TC, Wilson ML, Turner JE, et al. BacT/Alert: an automated colorimetric microbial detection system. J Clin Microbiol 1990; 28: 1608-1612.

11. Garner JS, Jarvis WR, Emori TG, Horan TC, Hughes JM: CDC definitions for nosocomial infections, 1988. Am J Infect Control 1988; 16: 128-140.

12. DeLong ER, DeLong DM, Clarke-Pearson DL: Comparing the areas under two or more correlated receiver operating characteristic curves: a nonparametric approach. Biometrics 1988; 44: 837-845.

13. Tokars JI: Predictive value of blood cultures positive for coagulase-negative staphylococci: implications for patient care and health care quality assurance. Clin Infect Dis 2004; 39: 333-341.

14. Khatib R, Riederer K, Saeed S, et al. Time to positivity in Staphylococcus aureus bacteremia: possible correlation with the source and outcome of infection. Clin Infect Dis 2005; 41: 594-598.

15. Kallio R, Surcel HM, Bloigu A, Syrjala H: C-reactive protein, procalcitonin and interleukin- 8 in the primary diagnosis of infections in cancer patients. Eur J Cancer 2000; 36: 889-894.

16. Nylen E, Muller B, Becker KL, Snider R: The future diagnostic role of procalcitonin levels: the need for improved sensitivity. Clin Infect Dis 2003; 36: 823-824.

17. Catton JA, Dobbins BM, Kite $P$, et al. In situ diagnosis of intravascular catheter-related bloodstream infection: a comparison of quantitative culture, differential time to positivity, and endoluminal brushing. Crit Care Med 2005; 33: 787-791.

18. Laffer RR, Frei R, Widmer AF: Epidemiology of septicemias in a university hospital over 5 years. Schweiz Med Wochenschr 2000; 130: 1471-1478.

19. Ghillani PP, Motte P, Troalen F, et al. Identification and measurement of calcitonin precursors in serum of patients with malignant diseases. Cancer Res 1989; 49: 6845-6851. 